Introduction The human intestinal tract is a complex microbial ecosystem unique to every individual and contributing sufficient metabolic equivalents to be considered an organ in its own right. Dysbiosis is thought to contribute to the chronic inflammation which is involved in colorectal cancer (CRC) development. We have shown that the premalignant adenoma stage of CRC is underpinned by chronic inflammation and hypothesise that the colonic microbiota is an important driver of neoplastic progression. The aim of the study was to compare bacterial diversity in adenomas and paired adjacent normal colonic tissue.

Methods 72 patients undergoing colonoscopy as part of the national bowel cancer screening programme were recruited. All patients underwent polypectomy for large adenomas $(>1 \mathrm{~cm})$. Denaturing gradient gel electrophoresis (DGGE on all subjects) and pyrosequencing (18 subjects) were used to compare the bacterial diversity present between the adenoma and adjacent normal paired tissue samples.

Results $46 \%$ of patients showed differences in microbial diversity by DGGE analysis between adenoma and adjacent normal tissue. $21 \%$ had only limited differences whilst $79 \%$ showed dramatically altered diversity profiles. Phylum-level comparisons across the study cohort, based on sequencing, revealed no statistically significant differences for the Bacteroidetes, Firmicutes and Proteobacteria phyla. When samples were split based on DGGE analysis (i.e. similar or different DGGE profiles between paired polyp and normal mucosa), eight of the possible 10 phyla showed statistically significant differences. Collectively samples with similar profiles between polyp and normal tissue (DGGE 'same' group) had higher levels of Bacteroidetes and lower numbers of Actinobacteria, Firmicutes and Proteobacteria than the DGGE 'different' group. However when genus-level comparisons were assessed the DGGE 'different' sample pairs demonstrated a much greater level of dysbiosis. The levels of normal commensal genera were reduced and there was evidence of large numbers of potentially pathogenic bacteria including Shigella, Enterobacter and Fusobacteria spp.

Conclusion Differences in microbial diversity harboured by a significant number of polyps compared to their immediately adjacent normal mucosa point to an important role for these bacteria in CRC progression. We hypothesise that the disturbed microbial homeostasis in these polyps, in genetically predisposed individuals and taking into account multiple environmental factors, underpins the pathogenesis of colorectal neoplasia. The identification of a "harmful" pro-inflammatory microbiota that is associated with precancerous stages (i.e. adenomas) offers the potential for manipulations of this microbiota with probiotic, prebiotic and indeed antibiotic means.

Disclosure of Interest None Declared.

\section{PWE-013 EVALUATION OF FAECAL ASPIRATE AS A PROXY FOR COLONOSCOPY BIOPSY SAMPLING TO ASSESS MICROBIAL DIVERSITY}

doi:10.1136/gutjpl-2013-304907.302

'E Watt, ' $S$ Berry, ${ }^{2} \mathrm{~F}$ Farquarson, 'M Glaire, ' $\mathrm{J}$ Thomson, ${ }^{1} \mathrm{E}$ El-Omar, ${ }^{2} \mathrm{P}$ Louis, 1."G Hold. ${ }^{1}$ Division of Applied Medicine; ${ }^{2}$ Rowett Institute of Nutrition and Health, Aberdeen University, Aberdeen, UK

Introduction The gut microbiota plays a key role in Inflammatory Bowel Disease (IBD) pathogenesis and is also involved in colorectal neoplastic progression. In particular, the "dysbiosis" theory has evolved from work which has shown IBD sufferers to have different microbiologic profiles to normal controls. There is strong evidence to demonstrate that individuals harbour a unique microbiota with faecal and mucosal ecosystems being distinct. This would indicate that faecal samples are not appropriate surrogate markers for mucosal samples and other alternative sampling options should be considered. The aim of the study was to assess the microbial diversity of paired biopsies and faecal aspirates to determine whether faecal aspirate can be used as a suitable surrogate for colonic biopsies.

Methods Bacterial DNA was extracted from 21 paired mucosal biopsies and faecal aspirates, and subjected to denaturing gradient gel electrophoresis (DGGE) and q-PCR analysis to quantify the relative abundance of the following major bacterial groups: Bacteroidetes, Ruminococcaceae, Lachnospiraceae and Enterobacteriaceae. The relative abundance of bacterial groups was expressed as a percentage of total bacteria.

Results Increased variation was found across the faecal aspirates compared to biopsies with regards to relative abundance of the four bacterial groups analysed by q-PCR (Table 1). This indicates that microbial diversity of faecal aspirates is more variable than mucosal biopsies. When the mean abundance of each bacterial group across the biopsies and across the faecal aspirates was compared using a Paired t-test, there was a significant difference in levels of Bacteroides (62.3\% vs $44.4 \% ; p=<0.05)$ but not the other bacterial groups. There was no statistical correlation between paired faecal aspirate and biopsy samples when analysed for the four major bacterial groups by linear regression.

\section{Abstract PWE-013 Table 1}

\begin{tabular}{|c|c|c|c|c|}
\hline & \multicolumn{2}{|l|}{ Biopsy } & \multicolumn{2}{|l|}{ Fecal aspirate } \\
\hline & $\begin{array}{l}\text { Abundance } \\
\text { across } \\
\text { samples* (\%) }\end{array}$ & Mean +/- SD & $\begin{array}{l}\text { Abundance } \\
\text { across } \\
\text { samples (\%) }\end{array}$ & Mean +/- SD \\
\hline Bacteroides & $40.5-116.3$ & $62.3 \% \pm 20.0 \%$ & $0.4-84.5$ & $44.4 \% \pm 25.8 \%$ \\
\hline Clostridium cluster XIVa & $11.8-63.7$ & $36.7 \% \pm 16.8 \%$ & $9.2-150.4$ & $39.4 \% \pm 37.5 \%$ \\
\hline Clostridium cluster IV & $3.5-23.4$ & $11.9 \% \pm 5.9 \%$ & $1.8-36.0$ & $11.9 \% \pm 9.6 \%$ \\
\hline Proteobacteria & $0.07-34.4$ & $5.6 \% \pm 8.4 \%$ & $<0.1-59.6$ & $4.0 \% \pm 13.1 \%$ \\
\hline
\end{tabular}

* $=$ calculated based on relative abundance as a percentage of total bacteria

Conclusion Substantial microbial diversity differences exist between faecal aspirate and biopsy samples. Faecal aspirates demonstrate considerably more variation than biopsies, and there would appear to be no correlation between paired samples. These results suggest that faecal aspirate is not a suitable microbiological surrogate for mucosal biopsies.

Disclosure of Interest None Declared.

\section{PWE-014 DEVELOPMENT OF AN OPTIMUM TREATMENT REGIMEN FOR TRANSCUTANEOUS POSTERIOR TIBIAL NERVE STIMULATION FOR FAECAL INCONTINENCE}

doi:10.1136/gutjnl-2013-304907.303

1,*G Thomas, 'T Dudding, 'R J Nicholls, 'C Vaizey. 'St Mark's Hospital and Academic Institute, London, UK

Introduction Posterior tibial nerve stimulation (PTNS) is an effective treatment for faecal incontinence (FI). A wide range of PTNS regimens have been reported. The aim of this study was to determine an improved PTNS regimen.

Methods Patients were randomised to either daily or twice weekly unilateral transcutaneous PTNS for six weeks. The investigator was blinded to group allocation. Once completed, further patients were recruited. They received daily bilateral transcutaneous PTNS for six weeks. The primary outcome measure was the change in frequency of FI episodes per week. PTNS was self-administered by each patient from home.

Results Thirty patients received either daily or twice weekly PTNS. The daily unilateral PTNS group achieved a reduction in frequency of incontinent episodes of 5(11.3) to 3.5(4.31), $\mathrm{p}=0.025$ Three achieved complete continence. No change was seen in the twice weekly group. Neither group improved deferral time. A further 20 patients received daily bilateral PTNS. They achieved a 